\title{
IN SITU MONITORING OF III-V PROCESSING
}

F. L. Terry, Jr.

Solid State Electronics Laboratory, Dept. of Electrical Engineering and Computer Science, University of Michigan, Ann Arbor, MI. 48109-2122, fredty@umich.edu

\begin{abstract}
Fabrication of high performance III-V devices and integrated circuits depends on careful control of layer thicknesses and compositions in the as-grown epitaxial layers and in the etching of these layers. The relatively high value of compound semiconductor devices (compared with high-volume Si devices) makes the use of advanced process control (with expensive in situ sensors) potentially advantageous. Considerable attention has been given to the problems of realtime feedback control of MBE growth systems. In this paper, I will discuss experiences with use of both in situ and ex situ monitors for controlling reactive ion etching (RIE) of III-V materials. Specific examples from an electron cyclotron resonance (ECR) RIE base contact etch from an AlInAs/GaInAs HBT process will be given. The relative merits of reflection-based wafer sensors vs. process state sensors (optical emission spectroscopy and mass spectroscopy) will be discussed. The unique opportunities and problems associated with the III-V materials and required etch processes will be contrasted to implementation of advanced wafer state endpoint detection schemes in $\mathrm{Si}$ and flat panel display processes. Specific problems and solutions from our research which I will discuss include chamber seasoning effects on the drift of optical emission based endpoint detection schemes and signal processing techniques for accounting for this drift, modeling of the optical dielectric function of the compounds of interest vs. composition, and the effects of surface roughness on optical thickness measurements.
\end{abstract}

\section{INTRODUCTION}

Advanced process control (APC) based on in situ and in line monitors (sensors) is being pursued by researchers in all aspects of thin-film technology (Si VLSI, compound semiconductor devices, flat panel displays, advanced optical coatings, etc.) to improve process margins with the coupled goals of improving the manufacturability of leading-edge devices and reducing costs. In compound semiconductor fabrication, in situ monitors and real-time feedback control have been relatively aggressively applied to epitaxial growth control for both MBE and OMCVD reactors. $^{1,2,3,4,5}$ This is a very sensible starting point, as the quality of the epitaxial layers is certainly the most critical factor in the manufacture of high performance compound semiconductor devices. However, many of these device structures also require careful control of either dry or wet chemical etches to expose layers for application of ohmic contacts and Schottky gates. Less attention has been paid to the problems of etch control in III-V compound materials. In this paper, I will address some of the issues and possible solutions for high-accuracy etch endpoint detection.

To effectively implement in situ sensor-based APC schemes, it is, of course, necessary to have the in situ sensors and related equipment controller hardware and software. Also, it is important to have available effective ex situ, in-line measurement systems. These systems are needed both to verify the effectiveness of the in situ based systems and to provide 
complementary measurements which cannot be performed in situ. In Si VLSI fabrication, rapid, nondestructive, in-line measurements are commonly used to monitor film thicknesses and linewidths on sampled product lots as part of routine statistical process control (SPC) schemes. In research and development, the Si VLSI industry is moving toward implementation of batchto-batch and wafer-to-wafer control (two variations of run-to-run control) by increasing the use of in-line metrology. In III-V device fabrication, the most common process control approach is to develop individual unit process using relatively simple test structures and to evaluate the processes using relatively sophisticated measurements including high resolution X-ray diffraction (rocking curves), photoluminescence, photoreflectance, etc; however, relatively few in-process measurements are typically performed on device wafer prior to electrical test. As one of the results of our process control efforts, I will illustrate the use of a common $\mathrm{Si}$ in-line metrology tool, the microscope-based spectral reflectometer, for use in compound semiconductor process monitoring.

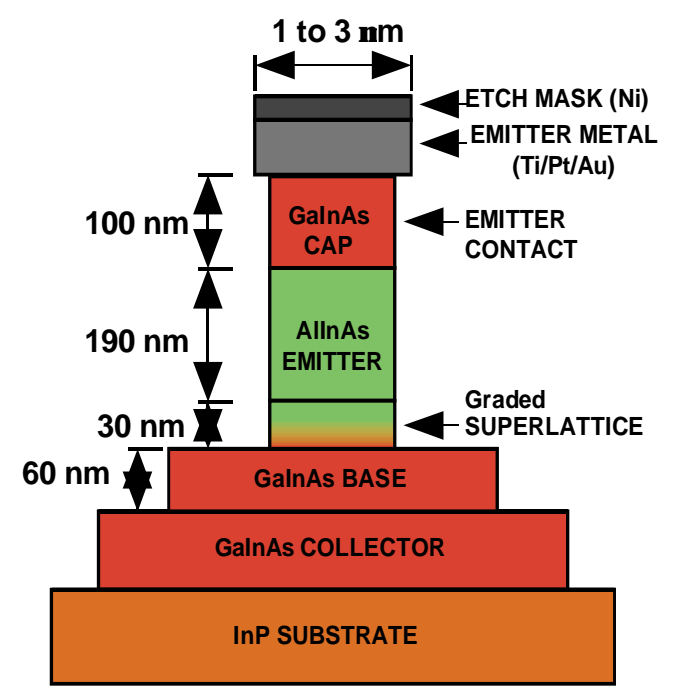

Figure 1 Schematic cross section of the HBT device which was the subject of this process control project.

The experimental results which I will discuss in this paper were primarily the result of an effort to control the dry etching of a self-aligned base contact etch in a Hughes Research Laboratories (HRL, Malibu, Ca.) InP-based heterojunction bipolar transistor process. ${ }^{6}$ The device cross-section is shown in Figure 1. The critical requirements for this process were to etch through the GaInAs cap, AlInAs emitter, the graded GaInAs/AlInAs superlattice (SL), and finally to stop in the GaInAs base with no more than $5 \mathrm{~nm}$ of over-etch. The accuracy with which this goal could be achieved would determine the minimum allowed base width and, thus, would be a major determining factor in the high frequency performance of the HBT. Also, since the base contact metallization was to be applied in a self-aligned approach, a controlled undercut of the emitter contact metal was also needed. Finally, the dry etch need to be performed in a low damage mode. The electron cyclotron resonance (ECR) dry etch process developed at the University of Michigan to address this problem has been documented elsewhere. ${ }^{7}$ In this paper, I will restrict the discussion to the successes and failures of the in situ and in-line sensors employed in the process control effort, and to a discussion of possible improved solutions for problems of this type. 


\section{EXPERIMENT}

The ECR-based dry etch system with the in situ monitors which we employed is illustrated schematically in Figure 2. The etch system was built by PlasmaTherm, Inc. and incorporate a $2.45 \mathrm{GHz}$ ECR source from Wavemat, Inc. The wafer chuck was mechanically-clamped and Hebackside cooled, and could be raised and lowered via a bellows arrangement to enable optimization of the wafer position relative to the ECR source. The wafer could be separately biased with a $13.56 \mathrm{MHz}$ RF source. The process used for all of the etch experiments described in this work used an $\mathrm{Cl}_{2} / \mathrm{Ar}$ chemistry. ${ }^{7}$

\section{IN SITU MONITORS}

To perform automated endpoint detection for the base contact etch, we installed three in situ monitoring devices

1. A differentially-pumped quadrupole mass spectrometer (QMS);

2. A monochromator-based optical emission spectrometer (OES);

3 . A dual- $\lambda$ reflectometer.

These systems are illustrated in Figure 2. The QMS was a MKS PPT system which uses an open source ionizer, $6.3 \mathrm{~mm}$ quadrupole rods, and an electron multiplier detector. Process gas was sampled into the QMS through a small port in the chamber near the typical level of the wafer. Due to the variable vertical position of the wafer chuck, it was not easily possible to place a sampling port for the QMS very close to the wafer itself during the etch.

The OES system was constructed using a Spex 500M 0.5 meter Czerny-Turner monochromator, a room temperature Hammamatsu R636 GaAs-photocathode photomultiplier tube (PMT), and a Stanford Research 570 low noise current preamplifier. A multi-stranded quartz optical fiber was used to bring light to the monochromator. The fiber bundle was arranged in vertical line and focused onto the entrance slit of the monochromator to maximize the signal strength. This arrangement was used (rather than a multi-wavelength array detector) to provide the maximum possible signal to noise ratio for a single emission species.

The reflectometer system (illustrated in Figure 3) was designed and constructed using a polarizer-photoelastic modulator (PEM)-polarizer system to provide high-frequency $(100 \mathrm{KHz})$ amplitude modulation of the probe beam and the reflected beam was demodulated using a pair of SR850 lockin amplifiers. This arrangement gave good signal to noise ratios using a $75 \mathrm{~W}$ Xe lamp source with the sample approximately $1 \mathrm{~m}$ from the beam-splitter. A lamp source was used rather than lasers to permit optimization of the probe wavelengths to the regions of maximum index differences between the layers of interest. There are relatively large differences in the refractive indices of AlInAs and GaInAs at $\mathrm{Hg}$ emission lines; therefore, we intended to perform the base endpoint experiment using a Hg lamp. The experimental performance of this system on a polycrystalline Si test sample and the theoretical performance on the HBT are shown in Figure 4. Two wavelengths were employed rather than the more common single wavelength arrangement (typically $\mathrm{HeNe}$ laser-632.8 $\mathrm{nm}$ ) to improve the sensitivity. As in the case of the OES system, discrete PMT detection was used to maximum the SNR at the expense of the additional information available from spectral measurements. The reflectometry system was deployed in a normal-incidence configuration due to the vertical movement of the wafer stage 
(which precluded the use of a pair of off-normal "ellipsometry" ports for specular reflection measurements).

The basic rational was that the QMS and OES systems would provide chemical process state information on the plasma environment and that the dual- $\lambda$ reflectometer would provide realtime information on the remaining film thickness during the etch. In principle, any one of the in situ monitors should have been able to detect the transition from the AlInAs layer to the GaInAs layer by sensing either the presence of $\mathrm{Ga}$ (for the two chemical process state sensors) or the change in the reflection (for the reflectometer). The apparent challenges for all three monitors lay in the speed and accuracy, and the problem of detecting the non-abrupt transition from the graded AlInAs/GaInAs SL into the GaInAs base layer.

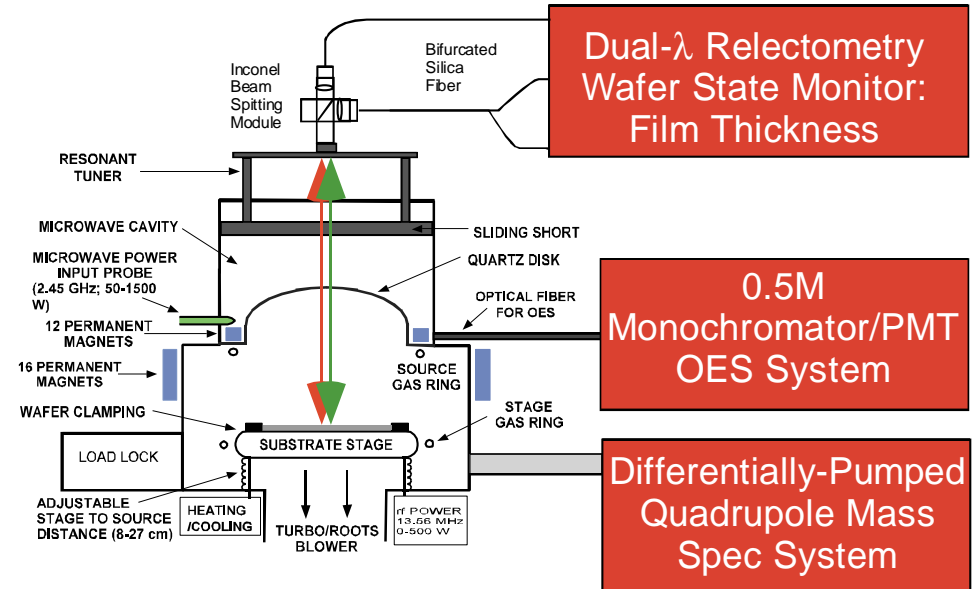

Figure 2 Illustration of ECR etch system used in the HBT control project and in situ monitors employed.

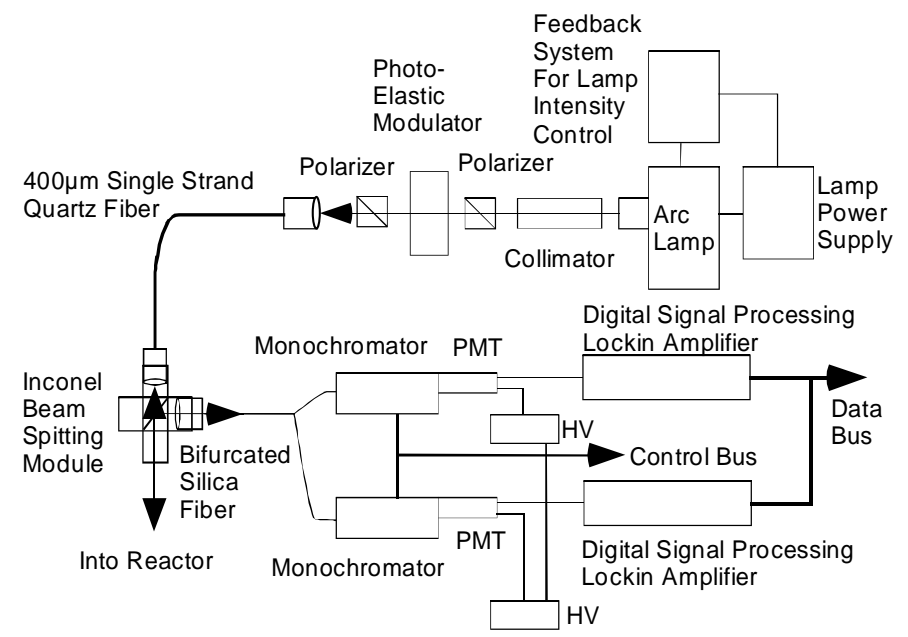

Figure 3 Cross-polarizer/PEM dual- $\lambda$ reflectometer for in situ film thickness monitoring. 


\section{IN-LINE MONITORS}

To provide routine film thickness measurements for evaluation the endpoint detect schemes, we modified the software of a commercial microscope-based spectral reflectometer system (a Leitz MPV-SP). The software was modified to allow arbitrary layer structures to be input into the optical modeling routines. The hardware itself was not modified. The system provided measurements of the sample reflectance vs. wavelength over the 400-800nm spectral range using a scanning monochromator-PMT arrangement. We also added a computer controlled scanning stage for late in this work. The measurement spot size could be varied from as small as $1 \mathrm{x} 1 \mu \mathrm{m}$ (using a 100X objective) to larger sizes. We typically used a 100x100 $\mu \mathrm{m}$ spot size delivered through a 10X objective. Newer systems from a variety of manufactures (KLA-Tencor, Nanometrics, Leica, and others) offer these capabilities without modification, but are not yet commonly used in compound semiconductor efforts. The use of a microscope-based reflectometer for ex situ film thickness measurement is advantageous both for mapping of sample uniformity over a wafer and for performing measurements in nominally uniform regions (away from contacts or other topography). Also, these measurements could be made at a time of only a few seconds per measurement spot.
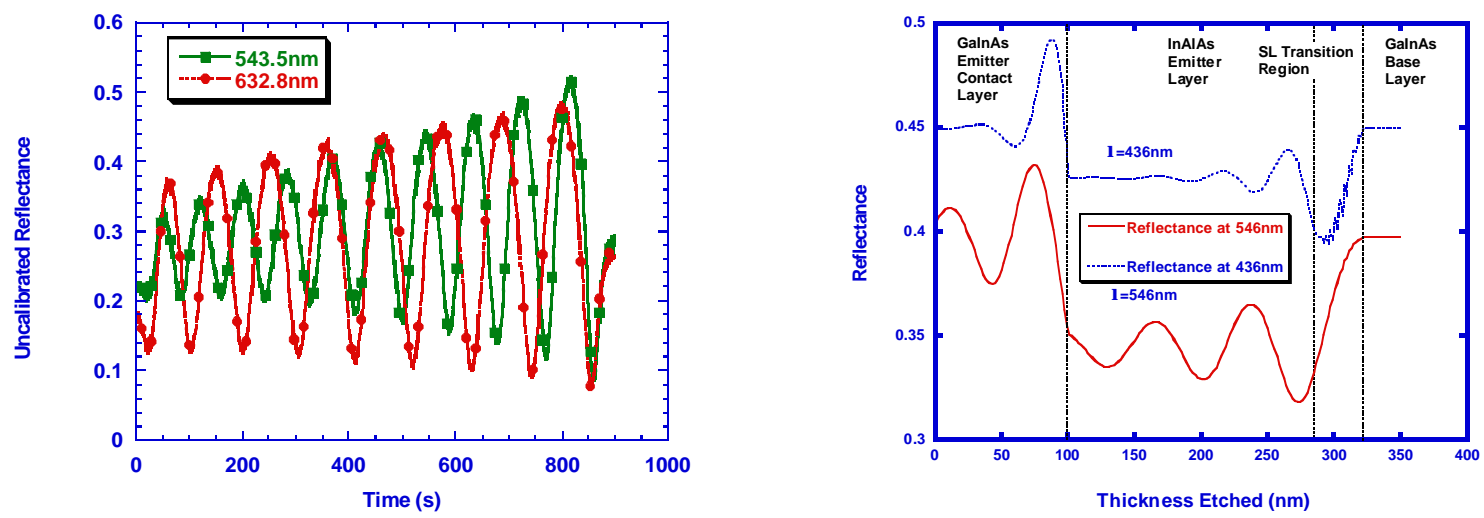

Figure 4 Experimentally measured response of dual- $\lambda$ reflectometer on a poly $\mathrm{Si} / \mathrm{SiO}_{2} / \mathrm{Si}$ test sample etch in a $\mathrm{Cl}_{2}$ environment in the ECR (left) and the theoretical response of the reflectometer for the AlInAs/GaInAs HBT structure. The experimental data was collected through the quartz dome in the ECR system with the sample approximately $1 \mathrm{~m}$ from the beam splitter. The data was collected at a $0.1 \mathrm{~s}$ sampling time. A $75 \mathrm{~W}$ Xe lamp was used as the light source.

To provide the reference data on the optical dielectric functions needed for operation of both the in situ reflectometer and the in-line spectral reflectometer, we characterized our materials and samples using ex situ spectroscopic ellipsometry using both a Rudolph S2000 system and a Sopra GESP-5 system. 


\section{RESULTS}

\section{MASS SPECTROSCOPY}

In our test experiments on GaAs wafers, the QMS did not show any Ga-containing species ${ }^{8,7}$ and was therefore not used in attempting to endpoint the HBT etch. At least one group has been successful on a similar attempt using a similar quadrupole system but with a sampling tube located near the wafer being etched. ${ }^{9}$ In our configuration, it is possible that the Ga-containing species were being removed by deposition reaction on the plumbing prior to the quadrupole mass filter itself or in the ionizer. In addition to the problem of the movable sample stage discussed earlier, we chose not to invest further effort into this sensor for two reasons. First, placing a sampling tube near the wafer (an thus with exposure to the plasma) introduces the possibility of significant wafer contamination. Also, due to the well-known problem of process gas induced gain shifts when using thoriated iridium filaments, we had attempted to use tungsten filaments in the ionizer. However, due to the $\mathrm{Cl}_{2}$ in our etch gas, the lifetime of the $\mathrm{W}$ filaments was too short for practical use. Thus, while the QMS initially seemed to be a straightforward solution, our conclusion was that it is not a practical solution for even limited lot production applications.

\section{OPTICAL EMISSION SPECTROSCOPY}

The OES system provided a usable endpoint signal from the Ga emission line at $417.2 \mathrm{~nm}$. At typical test run is illustrated in Figure 5. As can be seen in this figure, the major regions of the sample can be resolved and the approximate etch rates of each material can be estimated. However, high accuracy endpointing of the etch required a more sophisticated procedure than simply detect the presence of Ga at some predefined OES signal threshold The problem is that the chamber becomes seasoned with Ga-containing compounds after only a few runs, and, thus, there is a time-varying Ga OES signal present even while etching the AllnAs. By using a more sophisticated signal processing approach, we were able to demonstrate acceptably high accuracy endpoint detection on simple test samples with abrupt layers of AlInAs on GaInAs on InP. ${ }^{10}$ The maximum-likelihood estimator method described in this reference could be readily adapted to the grade SL case, but was not used due to limitations in available samples. The basic problem is that some level of experimental "training" of the algorithm was required for each type of sample and also this effort must be repeated on a roughly daily basis to correct for major etch system drifts. In production applications with repeated runs of the same type of product through an etch system, the combination of the OES method (interpreted through a drift correcting algorithm) and ex situ in-line metrology would provide a reliable solution. Without this repetition, the requirement for calibration runs would probably make this technique to expensive to implement.

\section{DUAL- $\lambda$ REFLECTOMETRY}

Although it was test successfully on Si test samples (see Figure 4), the dual- $\lambda$ reflectometer was not used successfully for endpointing the HBT etch due to rapid clouding of the ECR quartz dome. This system was successfully test in a poly $\mathrm{Si}$ endpoint experiment on a parallel plate RIE. The reflectometer signal was initially calibrated using a bare Si wafer in the chamber prior to the etch run. The signal was then calibrated for window changes and alignment errors by fitting the 
measured data to the theoretical reflectance a the last peak and valley of the reflectance oscillations to provide a gain and offset correction. The endpoint was then triggered when either wavelength signal reached the desired theoretical reflectance. A series of poly $\mathrm{Si} / \mathrm{SiO}_{2} / \mathrm{Si}$ runs resulted in an oxide over-etch of $3.6 \mathrm{~nm}$ with a standard deviation of only $0.87 \mathrm{~nm}$. The overetch could have easily been reduced by adjusting the target reflection value.

In related work on flat panel displays, we have shown that dual- $\lambda$ reflectometry coupled with advanced signal processing (Extended Kalman Filtering) can etch to desired target remaining film thicknesses to an accuracy of $1.5 \mathrm{~nm}(3 \sigma$ limit $) .{ }^{11}$ In these experiments, films of $\mathrm{a}-\mathrm{Si} / \mathrm{SiNx} / \mathrm{Ta} / 1737 \mathrm{f}$ display glass were etched to a target remaining film a-Si thickness of 50nm. Other target thicknesses could have been chosen. The remaining a-Si film thickness had average film thickness of $48.4 \mathrm{~nm}$ with an extreme spread in all experiments of $1.4 \mathrm{~nm}$ (as measured by ex situ spectroscopic ellipsometry).



Figure 5 Typical OES signal $(417.2 \mathrm{~nm})$ while etching completely through a test structure. The approximate positions of the layers on this signal are indicated. The conditions of this run were: $50 \mathrm{~W}$ microwave power, 100W RF power, 3sccm $\mathrm{Cl}_{2}, 27 \mathrm{sccm}$ Ar, 2 mTorr pressure.

\section{IN LINE MONITORS}

We characterized the optical properties of the materials in the HBT structure using ex situ spectroscopic ellipsometry. Most the effort required was in the characterization of the AlInAs, a material which previously had not been extensively studied for optical properties in the UV-VisNIR range. ${ }^{12}$ This data was then used in our ex situ film thickness monitoring efforts. A spectroscopic ellipsometry (SE) measurement of an HBT test sample is shown in Figure 6 and a microscope-based spectral reflectometry (SR) measurement of the same sample is shown in Figure 7. As can be seen in the figures, the agreement between the two methods is good. As expected, the SE result has better statistical confidence limits due to the inherently higher accuracy of this optical measurement; however, the SR measurement were sufficiently accurate for our process monitoring applications and could be made in much smaller areas. 


\begin{tabular}{|c|c|}
\hline$\frac{\text { Growth }}{\text { Target }}$ & $\begin{array}{c}\text { SE } \\
\text { Analysis }\end{array}$ \\
\hline & $\begin{array}{l}1.88 \pm 0.03 \mathrm{~nm} \\
\text { Oxide }\end{array}$ \\
\hline $\begin{array}{l}\text { 100nm } \\
\text { InGaAs }\end{array}$ & $\begin{array}{l}94.41 \pm 0.16 \mathrm{~nm} \\
\text { InGaAs }\end{array}$ \\
\hline $\begin{array}{l}\text { 190nm } \\
\text { InAlAs }\end{array}$ & $\begin{array}{l}171.81 \pm 0.29 \mathrm{~nm} \\
\text { InAlAs }\end{array}$ \\
\hline $\begin{array}{l}9 \text { Period } \\
\text { InAIAs/InGaAs } \\
\text { Super Lattice } \\
\text { Period 3.33nm }\end{array}$ & $\begin{array}{l}\text { Simulated Using } \\
\text { Growth Targets }\end{array}$ \\
\hline $\begin{array}{l}\text { 60nm } \\
\text { InGaAs }\end{array}$ & $\begin{array}{l}61.64 \pm 0.40 \mathrm{~nm} \\
\text { InGaAs }\end{array}$ \\
\hline \multicolumn{2}{|c|}{ InP Substrate } \\
\hline
\end{tabular}

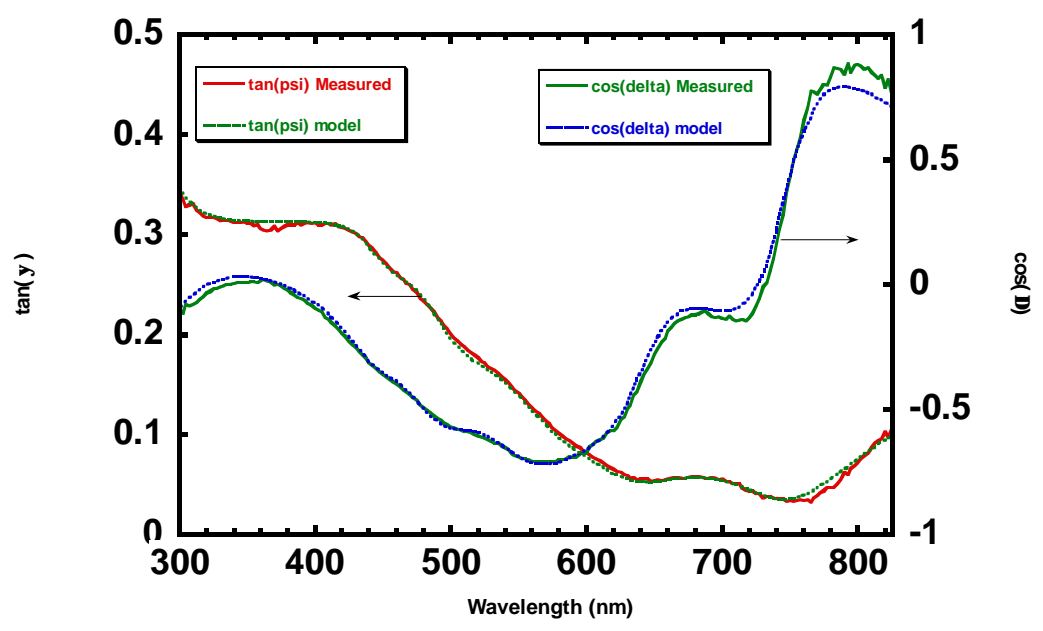

Figure 6 Ex situ spectroscopic ellipsometry measurements of HBT test structure.

\begin{tabular}{|c|c|}
\hline$\frac{\text { Growth }}{\text { Target }}$ & $\begin{array}{c}\underline{\mathrm{SE}} \\
\text { Analysis }\end{array}$ \\
\hline & $\begin{array}{l}0.93 \pm 0.99 \mathrm{~nm} \\
\text { Oxide }\end{array}$ \\
\hline $\begin{array}{l}\text { 100nm } \\
\text { InGaAs }\end{array}$ & $\begin{array}{l}96.97 \pm 1.16 \mathrm{~nm} \\
\text { InGaAs }\end{array}$ \\
\hline $\begin{array}{l}\text { 190nm } \\
\text { InAIAs }\end{array}$ & $\begin{array}{l}176.12 \pm 0.98 \mathrm{~nm} \\
\text { InAlAs }\end{array}$ \\
\hline $\begin{array}{l}9 \text { Period } \\
\text { InAIAs/InGaAs } \\
\text { Super Lattice } \\
\text { Period 3.33nm }\end{array}$ & $\begin{array}{l}\text { Simulated Using } \\
\text { Growth Targets }\end{array}$ \\
\hline $\begin{array}{l}\text { 60nm } \\
\text { InGaAs }\end{array}$ & $\begin{array}{l}60.22 \pm 2.87 \mathrm{~nm} \\
\text { InGaAs }\end{array}$ \\
\hline \multicolumn{2}{|c|}{ InP Substrate } \\
\hline
\end{tabular}

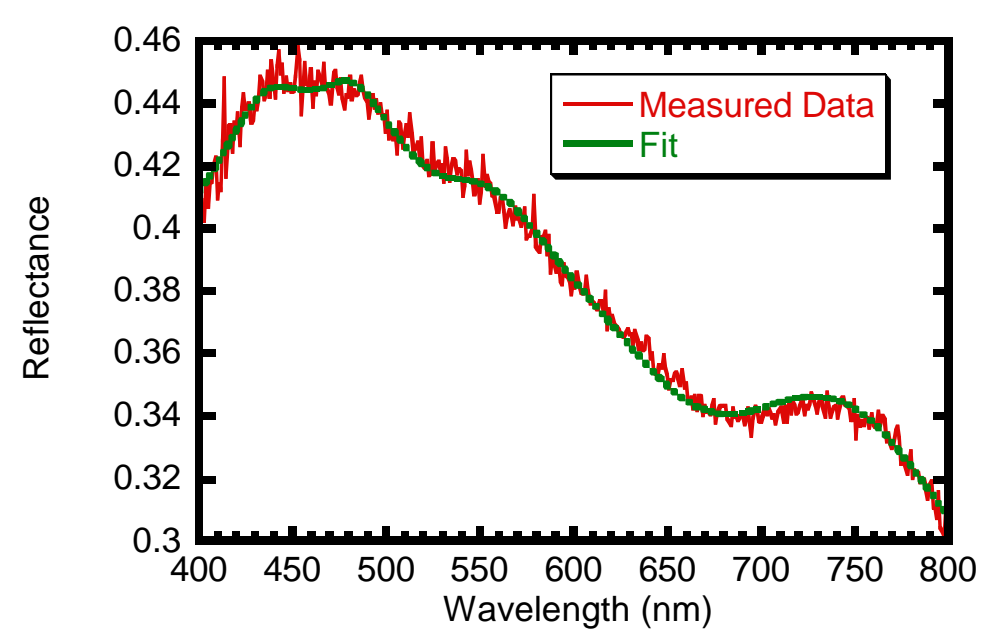

Figure 7 Microscope-based spectral reflectometry measurements of same test structure wafer as in Figure 7.

The relatively rapid SR measurement were used to quantify the accuracy of our OES based endpoint experiments. A before- and after-etch pair of measurements from a test sample are shown in Figure 8. However, two significant complications were noted. First, the apparent roughness layer on the sample was reduced following a DI $\mathrm{H}_{2} \mathrm{O}$ rinse of the sample. XPS results indicated that this rinse removed a Cl-containing film from the surface. ${ }^{7}$ Second, because diffuse scattering from surface rough layers has a more serious effect on SR measurements (which use absolute intensity) than SE measurements, we employed an algorithm adapted from BeckmannKirchoff scattering theory to model of the surface roughness layer. ${ }^{13}$ 
We also used the SR method to test the uniformity of the samples used in our endpoint experiments. We typically tested only 5 points per sample unless significant nonuniformities were detected. However, as an illustration of the results that are possible from a large number of measurements using an automated stage, a relatively nonuniform epi test sample is shown in Figure 9.
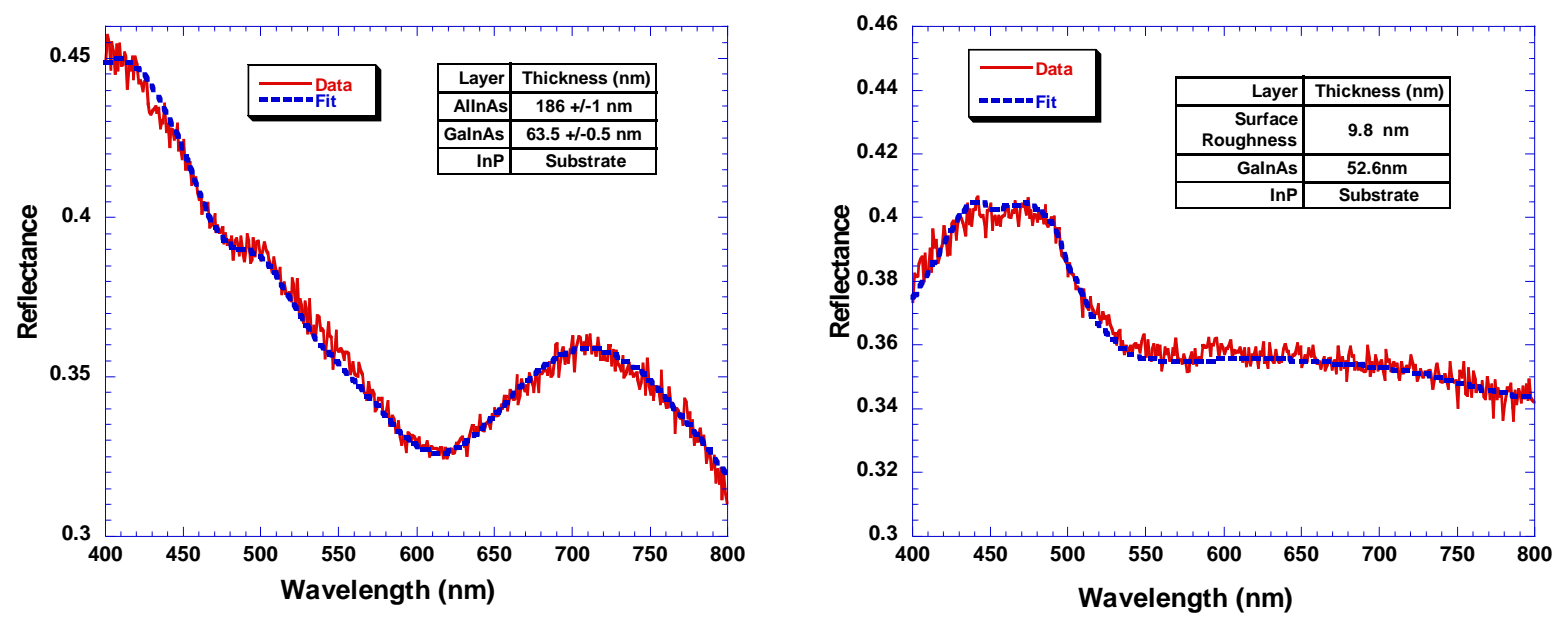

Figure 8 An abrupt interface AlInAs/GaInAs/InP test sample measured before and after etch using the microscope based SR method.

While the SE and SR characterization methods were successfully applied to ex situ sample measurements, we did identify a measurement problem which remains unsolved and presents a serious limitation to the wide-spread application reflectance measurement for monitoring of product (device) wafers. The problem is the accurate modeling of superlattices with very thin layers. As an example, a measurement and modeled SE response for the graded SL of the HBT structure is shown in Figure 8. We were unsuccessful in the fitting this structure with any of the models which we tried, including treating each layer as "bulk" materials (as in the figure), effective media approximations, quaternary alloy approximations (using harmonic oscillator interpolations), and others. In some respects this failure is not surprising as there is (intentionally) a significant amount of electron transfer between the layers in this type of structure. It might be expected that this sort of material would act as an anisotropic layer with unique optical properties.

\section{CONCLUSIONS}

While our attempts to accurately endpoint the targeted HBT fabrication step were not fully successful, I would like to offer the following conclusions and conjectures.

First, reflectance based techniques are capable of achieving few-monolayer level accuracies provided: (1) that there is an adequate optically clear view of the wafer surface; (2) that the optical properties of the materials are well characterized. Optical access to the wafer can be maintained in high density plasma systems if the sample positioning is fixed and off-normal ports are installed outside of the plasma discharge area. We are currently operating an in situ spectroscopic ellipsometry system on a high density, inductively coupled plasma tool (a Lam $9400 \mathrm{TCP}$ ) using $\mathrm{Cl}_{2} / \mathrm{HBr}$ etch chemistries. While the quartz plate below the TCP coil is heavily clouded by ion bombardment, chemical etching, and coating by etch byproducts, the ellipsometry ports have not shown any clouding or etching. Thus, this annoying problem can be 
readily solved once the geometry of the etch system is held fixed. In most cases, the optical properties of the materials of interest can be obtained by ex situ spectroscopic ellipsometry. Preparing and characterizing the specialized test samples required, however, often proves to be outside the financial scope of many projects. Our efforts to characterize AlInAs, while sufficient for this effort, were not complete. Significant effort is needed in the optical characterization and modeling of superlattice materials. This continues to be a limiting issue in the use of SE, SR, and related methods in both in situ and ex situ process monitoring efforts. Also, additional effort needs to be made in the optical modeling of process-induced damaged layer and etch byproduct layers. For all of these optical modeling complications, individual process endpoint solutions could be found by empirically matching the raw optical data to a known good shape; however, this type of solution is not robust against changes in sample layers structures or to changes in process-induced surface layers.

Second, process state measurements are well suited to control of repeated processes with significant product flow. However, for small lot applications, the effort and samples required in calibration and training of algorithms tends to offset their advantages. In these applications, SPC and run-to-run control methods using in-line monitoring tools may be more cost-effective. If runto-run variations in the process are too severe to allow effective adjustments using ex situ, in-line methods, then properly installed in situ optical metrology should prove effective.

Third, even if in situ monitors are available and effective, the use of microscope-based SR (a widely used tool in the Si industry) can be effectively employed for compound semiconductor applications. In particular, mapping of the thicknesses and uniformity of epitaxial layers would be useful in both not processing improperly grown wafers and in planning subsequent processing to account for variations in usable wafers. Depending on the layer thicknesses and absorption lengths, accurate estimates for the top two to four layers should be possible.

\section{ACKNOWLEDGEMENTS}

The author would like to thank Drs. S. Pang, W. Stanchina, J. Grizzle, P. Khargonekar, L. Kamlet, S. Thomas, III, C. Hanish, T. Vincent, T. Benson, and P. Bhattacharya, for helpful discussions and the use of some the data in this paper. He would also like to thank the following sponsors for their support of some of this work: DARPA Smart ECR Plasma Etching Program (F33615-92-C-5972); DARPA/AFOSR Center for Intelligent Electronics Manufacturing (F49620-95-1-0524); NSF Sensor-Based Control of Semiconductor Manufacturing Equipment Grant. (EID-9212316); State of Michigan Center for Display Technology and Manufacturing. 


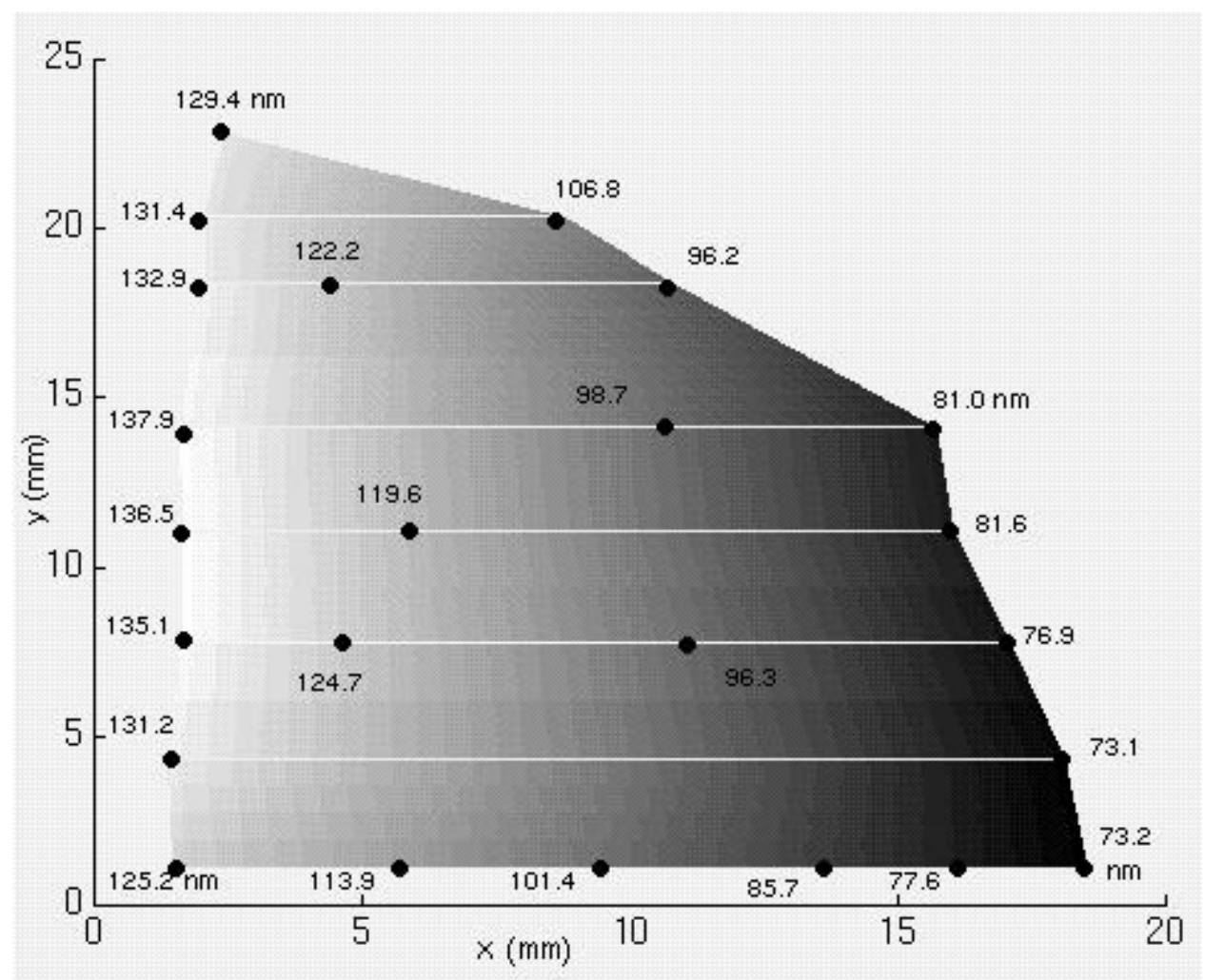

Figure 9 An SR measurement of the thickness uniformity of a $\mathbf{A l}_{0.44} \mathbf{I n}_{0.56} \mathrm{As} / \mathrm{InP}$ test sample.

\begin{tabular}{|c|}
\hline $\begin{array}{c}9 \text { Period } \\
\text { InAlAs/InGaAs } \\
\text { Super Lattice } \\
\text { Period 3.33nm }\end{array}$ \\
\hline $\begin{array}{c}70 \mathrm{~nm} \\
\text { InGaAs }\end{array}$ \\
\hline InP Substrate \\
\hline
\end{tabular}

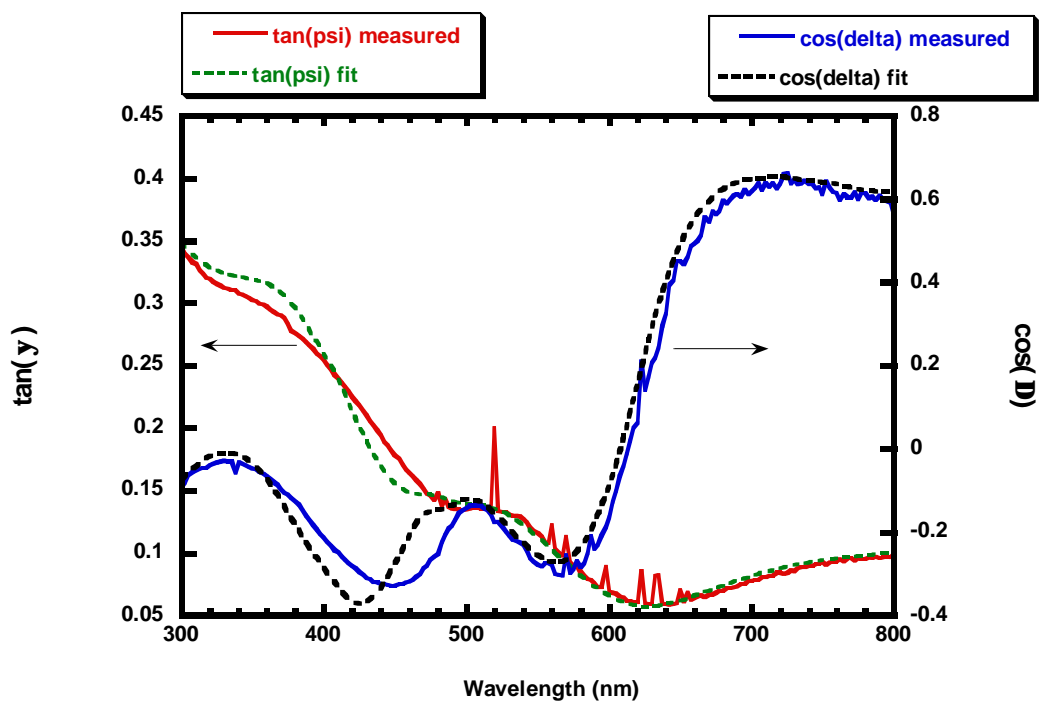

Figure $10 \mathrm{SE}$ measurement (right) of a graded superlattice test structure (left) and the modeled result from the growth target layer thicknesses. 


\section{REFERENCES}

${ }^{1}$ D. E. Aspnes, W. E. Quinn, M. C. Tamargo, M. A. A. Pudensi, S. A. Schwarz, M.J.S.P. Brasil, R. E. Nahory, S. Gregory, Appl. Phys. Lett., 60, pp.1244-6 (1992)

${ }^{2}$ G. N. Maracas, C. H. Kuo, S. Anand-S, R. Droopad, G.R.L. Sohie, T. Levola J. Vac. Sci. Techn, A13; pp.727-32 (1995).

${ }^{3}$ F.G. Celli, Y. C. Kao, T. S. Moise, M. Woolsey, T.B. Harton, K. Haberman, Diagnostic

Techniques for Semiconductor Materials Processing, Mater. Res. Soc, Pittsburgh, PA, USA; $\mathrm{xv}+585$ pp.365-70 (1996).

${ }^{4}$ J.D. Benson, A.B. Cornfeld, M. Martinka, K.M. Singley, Z. Derzko, P.J. Shorten, J.H. Dinan, P.R. Boyd, F.C. Wolfgram, B. Johs, P. He, J.A. Woollam, J. Electron. Mat. 25, pp.1406-10 (1996)

${ }^{5}$ C. Pickering, R. T. Carline, D.A.O. Hope, D.J. Robbins, Physica Status Solidi, A152; pp.95-102 (1995)

${ }^{6}$ M. Hafizi, W.E. Stanchina, R.A. Metzger, J.F. Jensen, F. Williams, IEEE Transactions on Electron Devices, 40, pp.2178-85 (1993).

${ }^{7}$ S. Thomas, III., Ph.D. Dissertation, University of Michigan, 1997, and references therein. ${ }^{8}$ S. Thomas, III, H. H. Chen, C. K. Hanish, J. W. Grizzle, S. W. Pang, J. Vac. Sci. Techn.. B14, pp.2531-6 (1996). ${ }^{9}$ D. L. Delville, J Budinavicius, D. A. Thompson, J. G. Simmons, Nuclear Instruments Methodsin Physics Research,-B106; pp.179-82 (1995)

${ }^{10}$ C.K. Hanish, J. W. Grizzle, H.H. Chen, L.I. Kamlet, S. Thomas, III, F. L. Terry, Jr., and S. W. Pang, J. Electron. Mat., 26, pp. 1401-8 (1997).

${ }^{11}$ T. L. Vincent, P.P. Khargonekar, and F. L. Terry, Jr., SID/IEEE International Display Research Conference Digest, Toronto, Canada, September 15-19, 1997, pp. $274-7$ (1997).

${ }^{12}$ L. I. Kamlet and F. L. Terry, Jr., Thin Solid Films, 313-4, pp. 435-441 (1998).

${ }^{13}$ T. E. Benson, A. Ramamoorthy, L. I. Kamlet, and F. L. Terry, Jr., Thin Solid Films, 313-4, pp. 177-182 (1998). 